\title{
A TR ESR STUDY OF THE QUENCHING OF PHOTOEXCITED DIOXOURANIUM (VI) SALTS BY STABLE NITROXYL FREE RADICALS
}

\author{
I. V. KHUDYAKOV and N. J. TURRO* \\ Department of Chemistry, Columbia University, New York, NY 10027, U.S.A.
}

Received 18 October 1992; accepted 3 November 1992

\begin{abstract}
TR ESR spectroscopy was applied to the study of the quenching of excited dioxouranium (VI) (uranyl) nitrate and sulfate by stable nitroxyl radicals of the 2,2,6,6-tetramethylpiperidine-1-oxyl (TEMPO) family. Photoexcitation of uranyl in solutions of alcohols of moderate viscosity $(\eta=3-10 \mathrm{cP})$ in the presence of TEMPO leads to CIDEP signals of TEMPO due to a radical triplet pair mechanism (RTPM). Polarized nitroxyls were also observed in solutions of polyelectrolyte sodium poly(styrenesulfonate), NaPSS, in the presence of the nitroxyl with a positively charged trimethylammonium group. Photolysis of uranyl salts in solutions of alcohols leads to the generation of free radicals of alcohols. No CIDEP of these radicals was observed, distinguishing $\mathrm{UO}_{2}{ }^{2+*}$ from its organic analog, the triplet benzophenone. The probable reason for the lack of polarization in uranyl photoreduction reactions is the difficult access of free radicals to the $\mathrm{U}$ atom of the solvated radical $\mathrm{UO}_{2}{ }^{+}(\mathrm{V})$; this atom bears the unpaired electron. The role of polyelectrolytes in the enhancement of the quenching of excited states is discussed. Results are in agreement with the statement that photoexcited uranyl has a triplet multiplicity.
\end{abstract}

\section{INTRODUCTION}

Stable free radicals and, in particular, nitroxyls (r) are known to be effective quenchers of photoexcited states of organic molecules [1-5]. The quenching of excited triplet molecules by a radical $\mathbf{r}$ leads to a singlet molecule plus the radical spin-polarized $\mathbf{r}^{*}$ [2,3]. The study of TR ESR spectra of $r^{*}$ provides important information on the elementary act of quenching and on the role of magnetic interactions. The present work is devoted to the TR ESR study of the quenching of photoexcited dioxouranium (VI) (uranyl) salts by $\mathbf{r}$; benzophenone triplet quenching by $\mathbf{r}$ was also studied for purposes of comparison. 


\section{EXPERIMENTAL}

\section{Instruments}

A detailed TR ESR description of the instrument is given elsewhere [5,6]. The instrument consists of a Bruker ER $100 \mathrm{D}$ spectrometer, a PAR model 4402 digital boxcar integrator, a Quanta Ray DCR 2A Nd: YAG laser (third harmonic $\lambda_{\text {ex }} 355 \mathrm{~nm}, 8$ ns fwhm, typical laser energy $10-15 \mathrm{~mJ} /$ pulse at $20 \mathrm{~Hz}$ ), and a Bruker broad band preamplifier with response time of $60 \mathrm{~ns}$. Typical sampling gates were put at 100-500 ns. We present TR ESR spectra below specifying $\Delta \mathrm{t}=\mathrm{A}-\mathrm{B}, \mathrm{s}$, where $\mathrm{A}$ is the beginning of the sampling after the laser pulse, and B is the end of the sampling, and the B-A value is the sampling gate. The system's response times were $c a .200 \mathrm{~ns}$. The experiments were run with a flow system consisting of a flat, thin $(0.5 \mathrm{~mm})$ quartz cell. Flow rates were chosen in the range of $2-5 \mathrm{ml} / \mathrm{min}$. Steady state ESR spectra of $\mathbf{r}$ were recorded on a Bruker ESR 300 spectrometer as first derivative spectra.

\section{Reagents and Solutions}

$\mathrm{UO}_{2}\left(\mathrm{NO}_{3}\right)_{2} \cdot 6 \mathrm{H}_{2} \mathrm{O}$ (Strem), $\mathrm{UO}_{2} \mathrm{SO}_{4} \cdot 3 \mathrm{H}_{2} \mathrm{O}$ (Fisher), 2,2,6,6-tetramethylpiperidine-1-oxyl (TEMPO, Aldrich) were used as received. Solvents from Fisher were used as received: ethylene glycol, propane-1-ol ( $n$-PrOH), propane-2-ol ( $i$-PrOH), 2-methybutane-2-ol (tertamyl alcohol), cyclohexanol, and benzene. Binary mixtures of ethylene glycol $/ i$-PrOH $(80: 20 \mathrm{v} / \mathrm{v})$ as well as cyclohexanol $/ n-\mathrm{PrOH}(50: 50 \mathrm{v} / \mathrm{v})$ were also used. (An addition of $i$-PrOH to ethylene glycol allows dissolution of TEMPO in a high concentration, $c a .10^{-2}$ M). The general abbreviation of alcohol will be ROH. Benzophenone (BP), benzhydrol $\left(\mathrm{BPH}_{2}\right)$, benzylic acid (BA) (all from Aldrich) were purified according to standard procedures, i.e. by recrystallization from water-ethanol solutions and/or by sublimation under vacuum. Sodium poly(styrenesulfonate) of MW 70,000 and 500,000 (both from Polysciences) was used; polymer denoted as NaPSS. NaPSS was purified by precipitation from a concentrated aqueous solution by excess of 1,4-dioxane in the method similar to that described in [7]. The precipitate was washed with 1,4-dioxane and dried. Aqueous solutions of NaPSS were prepared by dissolution of a known amount of polymer; molar concentrations of NaPSS were estimated by the use of molecular weights presented above. 4-Methylammonium-2,2,6,6-tetramethylpiperidine-1-oxyl, iodide (TEMPO ${ }^{-}$) was purchased from Molecular Probes and was synthesized according to the following procedure: 4-Amino-2,2,6,6-tetramethylpiperidine-1-oxyl (Aldrich) was mixed with methyliodide in a DMF solution over sodium carbonate powder at $\sim 273 \mathrm{~K}$ for several hours. TEMPO ${ }^{+}$was separated and recrystallized from a water-methanol mixture; the yield was ca. 90\%. TEMPO $\mathrm{NO}_{3}^{-}$was prepared from $\mathrm{TEMPO}^{+} \mathrm{I}^{-}$by the addition of a stoichiometric amount of $\mathrm{AgNO}_{3}$ to an aqueous solution of $\mathrm{TEMPO}^{+} \mathrm{I}^{-}$; the precipitate of AgI was filtered. Solutions of TEMPO ${ }^{+}$and $\mathrm{TEMPO}^{+} \mathrm{NO}_{3}{ }^{-}$display the expected ESR signals (see below).

Sodium dodecylsulfate (SDS, Bio-Rad Laboratories) was dissolved in aqueous solutions in order to obtain the micelles' concentration of $\sim 5 \cdot 10^{-3} \mathrm{M}$. 
Viscosities $(\eta)$ of binary mixtures were obtained from reference [8].

All solutions were deoxygenated (or otherwise stated) by prolonged argon bubbling, and the experiments were performed at room temperature.

\section{RESULTS}

\section{Uranyl photolysis in alcohols}

Upon photoexcitation of uranyl in solutions containing hydrogen (electron) donors, we were searching for TR ESR spectra of the donors radicals. We observed no TR ESR signals under photolysis of uranyl nitrate $\left(5 \cdot 10^{-3}-0.1 \mathrm{M}\right)$ solutions in neat alcohols $(n$ $\mathrm{PrOH}, i$-PrOH, 2-methylbutane-2-ol, cyclohexanol), nor in binary mixtures of ethylene glycol $/ i-\mathrm{PrOH}$ and cyclohexanol $/ n-\mathrm{PrOH}$, water $/ i-\mathrm{PrOH}$, water $/ n-\mathrm{PrOH}$ :

$$
\mathrm{UO}_{2}\left(\mathrm{NO}_{3}\right)_{2} \underset{\begin{array}{l}
\text { neat } \mathrm{ROH} \text { or } \\
\text { water } / \mathrm{ROH}
\end{array}}{\rightarrow} \text { No CIDEP }
$$

Addition of TEMPO $\left(1 \cdot 10^{-2}-4 \cdot 10^{-2} \mathrm{M}\right)$ to the solutions under investigation leads to TR ESR signals of polarized TEMPO only in solvents of moderate viscosity, namely 2methylbutane-2-ol, ethylene glycol $/ i-\mathrm{PrOH}$ and cyclohexanol $/ n-\mathrm{PrOH}(3 \leq \eta \leq 10 \mathrm{cP})$, see Figures 1 and 2.

$$
\begin{aligned}
\mathrm{UO}_{2}\left(\mathrm{NO}_{3}\right)_{2} & \multicolumn{2}{c}{\underset{\mathrm{ROH}, 3 \leq \eta \leq 10 \mathrm{cP},}{\mathrm{TEMPO}, 10-40 \mathrm{mM}}}
\end{aligned} \text { TEMPO }^{\#}
$$

ESR spectra of TEMPO and its derivatives are characterized by g-factors of $2.006 \pm$ 0.001 and hyperfine coupling constants $\mathrm{a}_{\mathrm{N}}$ of $15-17 \mathrm{G}$ depending upon the solvent and structure of the radical [9], cf. Figure 1 and 2. The use of lower concentrations of TEMPO $\left(5 \cdot 10^{-3}-1 \cdot 10^{-2} \mathrm{M}\right)$ in an ethylene glycol $/ i-\mathrm{PrOH}$ mixture led to the appearance of signals of radicals from alcohols as well as from TEMPO ${ }^{\sharp}$, see Figure 3.

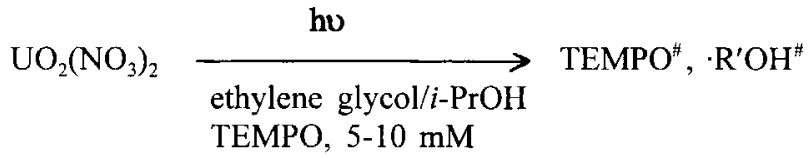




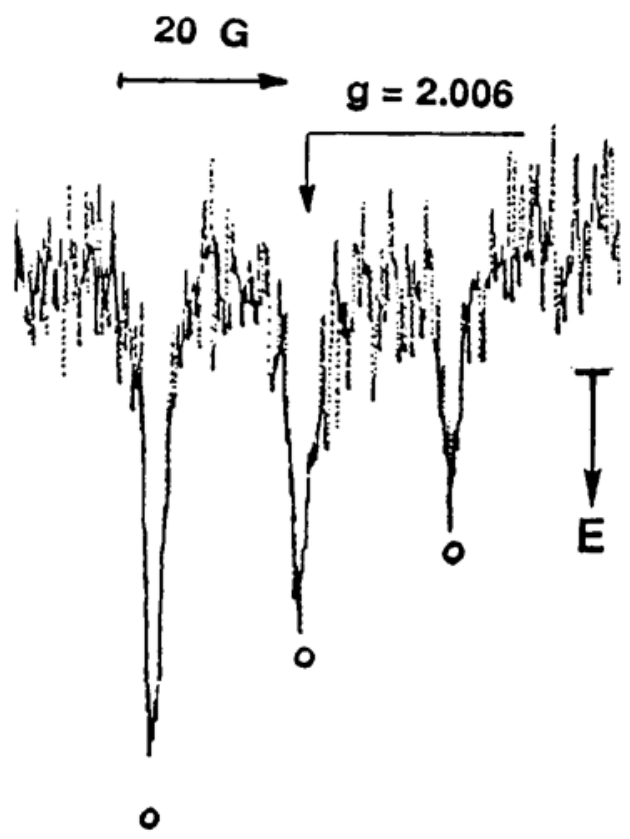

Figure 1. TR ESR spectrum obtained under photolysis of uranyl nitrate $(0.1 \mathrm{M})$ in the presence of TEMPO $\left(4 \cdot 10^{-2} \mathrm{M}\right)$ in ethylene glycol $/ i-\operatorname{PrOH}(80 ; 20 \% \mathrm{v} / \mathrm{v})$ binary mixture; $\Delta t=300-600 \mathrm{~ns}$. The circles stand for three TEMPO components.

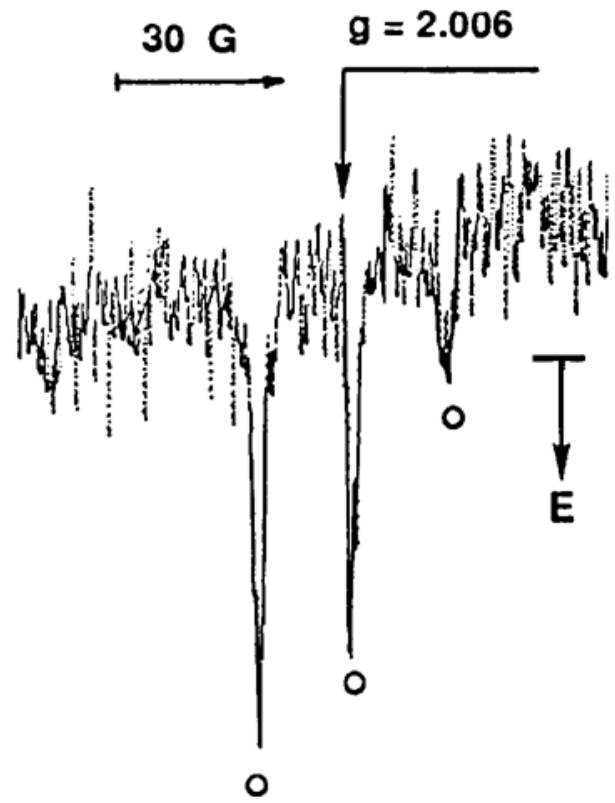

Figure 2. TR ESR spectrum obtained under photolysis of uranyl nitrate $(0.1 \mathrm{M})$ in the presence of TEMPO $\left(4 \cdot 10^{-2} \mathrm{M}\right)$ in cyclohexanol $/ n-\mathrm{PrOH}(50: 50 \% \mathrm{v} / \mathrm{v})$ binary mixture; $\Delta \mathrm{t}=300-600 \mathrm{~ns}$. The circles stand for three TEMPO components. 


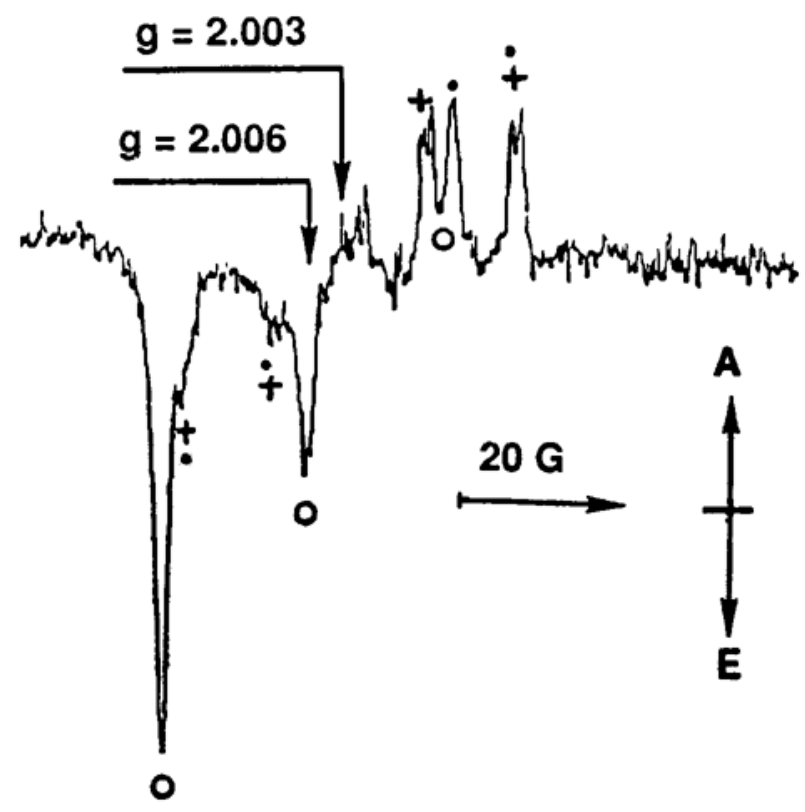

Figure 3. TR ESR spectrum obtained under photolysis of uranyl nitrate $(0.1 \mathrm{M})$ in the presence of TEMPO $\left(5 \cdot 10^{-3} \mathrm{M}\right)$ in ethylene glycol $/ i-\mathrm{PrOH}(80: 20 \% \mathrm{v} / \mathrm{v})$ binary mixture; $\Delta \mathrm{t}=0.7-1.0 \mu \mathrm{s}$. The circles stand for three TEMPO components; daggers stand for $\mathrm{CH}_{2} \mathrm{OHCH} \cdot \mathrm{OH}^{\#}$; and points, for $\left(\mathrm{CH}_{3}\right)_{2} \mathrm{C} \cdot \mathrm{OH}^{*}$ components, respectively.

(The assignment of components in the ESR spectrum of radicals from alcohols will be explained below in the Discussion section).

A TR ESR study of uranyl nitrate in SDS and NaPSS solutions

TR ESR of uranyl salts was studied in two microheterogeneous environments, i.e., in SDS micelles and in NaPSS solutions. Photolysis of uranyl nitrate or uranyl sulfate solutions $\left(5 \cdot 10^{-3}-10^{-2} \mathrm{M}\right)$ in SDS in the presence or absence of $\mathrm{BPH}_{2}$ or BA $\left(5 \cdot 10^{-3}-\right.$ $7 \cdot 10^{-3} \mathrm{M}$ ) did not lead to any TR ESR signals.

$$
\begin{aligned}
& \text { ho } \\
& \mathrm{UO}_{2}\left(\mathrm{NO}_{3}\right)_{2} \underset{\mathrm{SDS}, \mathrm{BPH}_{2} \text { or } \mathrm{BA}}{\longrightarrow} \text { No CIDEP }
\end{aligned}
$$

We also did not observe signals of TEMPO Tor TEMPO $^{+\#}$ ) under photolysis of the SDS solutions of uranyl salts in the presence of TEMPO $\left(5 \cdot 10^{-2}-10^{-2} \mathrm{M}\right)$ or $\mathrm{TEMPO}^{+} \mathrm{I}^{-}$or $\mathrm{TEMPO}^{+} \mathrm{NO}_{3}^{-}\left(\left[\mathrm{TEMPO}^{+}\right]=5 \cdot 10^{-3} \mathrm{M}\right)$. 


$$
\mathrm{UO}_{2}\left(\mathrm{NO}_{3}\right)_{2} \underset{\mathrm{SDS}, \mathrm{TEMPO}^{(+)}}{\stackrel{\mathrm{hu}}{\longrightarrow}} \text { No CIDEP }
$$

In experiments with NaPSS the polyelectrolyte was dissolved in aqueous solutions with concentrations of $1 \cdot 10^{-6}-5 \cdot 10^{-6} \mathrm{M}\left(\mathrm{MW} \mathrm{500,000)}\right.$ and $7 \cdot 10^{-6}-3.5 \cdot 10^{-5} \mathrm{M}$ (MW $70,000)$. The calculated concentration of monomer units in such solutions of NaPSS of both molecular weights was $2.5 \cdot 10^{-3}-1.2 \cdot 10^{-2} \mathrm{M}$. Photoexcitation of uranyl nitrate $\left(5 \cdot 10^{-3}\right.$ - $5 \cdot 10^{-2} \mathrm{M}$ ) in NaPSS solutions does not lead to any TR ESR signals. The nitrate or iodide of $\mathrm{TEMPO}^{+}$was dissolved in such solutions in concentrations of $5 \cdot 10^{-3}-2 \cdot 10^{-2} \mathrm{M}$. CW ESR spectra of aqueous NaPSS solutions of $\mathrm{TEMPO}^{+}$are characterized by $\mathrm{g}=2.005$ and $\mathrm{a}_{\mathrm{N}}=16.2 \mathrm{G}$. Laser photolysis of uranyl nitrate $\left(5 \cdot 10^{-3}-1 \cdot 10^{-2} \mathrm{M}\right)$ in the presence of TEMPO $^{+}(5-10 \mathrm{mM})$ led to emissive TR ESR signals of TEMPO ${ }^{\ddagger \sharp}$, see Figure 4.

$$
\mathrm{UO}_{2}\left(\mathrm{NO}_{3}\right)_{2} \underset{\text { NaPSS, TEMPO }}{\stackrel{\text { hu }}{\longrightarrow}} \mathrm{TEMPO}^{\prime \#}
$$

No significant difference was found in the intensities of the signals of iodide or nitrate $\mathrm{TEMPO}^{+}$under the same concentration of $\mathrm{TEMPO}^{+}$, as well as in solutions of a similar "monomer concentration" prepared by the dissolution of NaPSS of two different molecular weights.

A very weak TEMPO $^{\text {"\# }}$ signal was observed during the use of uranyl nitrate in a concentration of $1 \cdot 10^{-2} \mathrm{M}$. No observation was found of any TR ESR signals in solutions of $\left[\mathrm{UO}_{2}{ }^{2+}\right]>1.10^{-2} \mathrm{M}$.

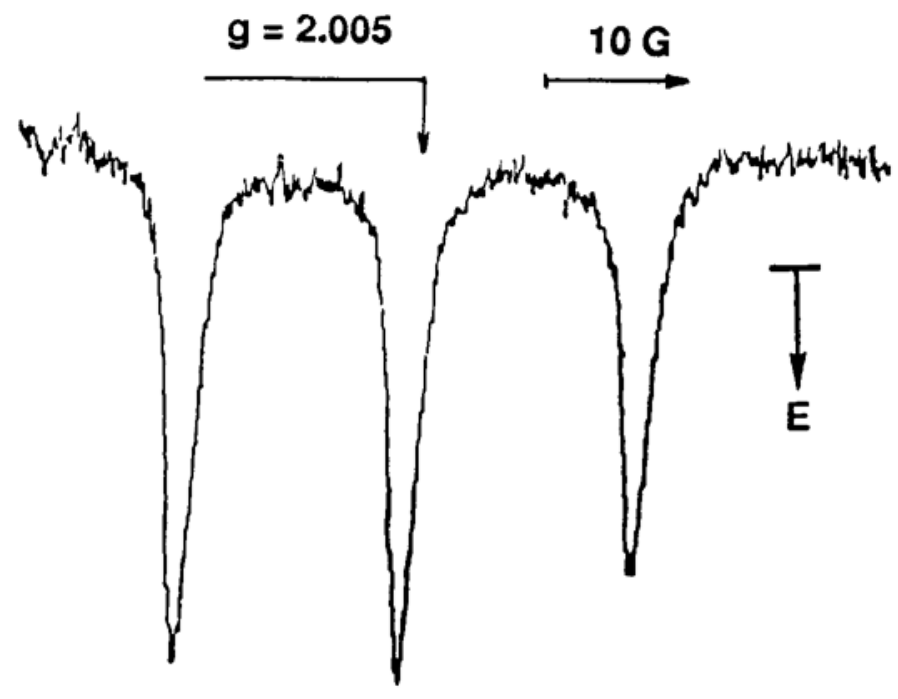

Figure 4. TR ESR spectrum obtained under photolysis of uranyl nitrate $\left(3 \cdot 10^{-3} \mathrm{M}\right)$ in the presence of TEMPO$^{+} \mathrm{I}^{-}\left(1.5 \cdot 10^{-3} \mathrm{M}\right.$ ) in NaPSS (MW 500,000, $\left.2.5 \cdot 10^{-6} \mathrm{M}\right) ; \Delta \mathrm{t}=300-600 \mathrm{~ns}$. 
TR ESR study of solutions of BP

Photoexcitation of BP $\left(5 \cdot 10^{-3}-1 \cdot 10^{-2} \mathrm{M}\right)$ in the alcohols listed above leads to TR ESR signals of a benzophenone ketyl-free radical, $\mathrm{BPH}^{*}$ and polarized free radicals of alcohol, namely $\left(\mathrm{CH}_{3}\right)_{2} \mathrm{C} \cdot \mathrm{OH}^{\#}$ from $i-\mathrm{PrOH}, \mathrm{CH}_{3} \mathrm{CH}_{2} \mathrm{CH} \cdot \mathrm{OH}^{\#}$ from $n-\mathrm{PrOH}$, etc., (see Figures 5 and 6), Eq. (7).

$$
{ }^{3} \mathrm{BP}^{*}+\mathrm{ROH} \rightarrow \mathrm{BPH}^{*}+\cdot \mathrm{R}^{\prime} \mathrm{OH} \cdot{ }^{*}
$$

where $\cdot \mathrm{R}^{\prime} \mathrm{OH}$ is an $\alpha$-hydroxyalkyl free radical of the alcohol.

The assignment of lines in the TR ESR spectra (Figures 5 and 6) is based on the data in reference [9]; in particular, the TR ESR spectra of $\mathrm{BPH}^{*}$ and $\left(\mathrm{CH}_{3}\right)_{2} \mathrm{C}^{-\mathrm{OH}^{*}}$ radicals (Figure 5) are in agreement with the corresponding spectrum presented in reference [10]. Figure 5 shows a spectrum with additional lines of the $\mathrm{CH}_{2}(\mathrm{OH}) \mathrm{CH} \cdot \mathrm{OH}^{\#}$ radical from ethylene glycol. These components are observed under photolysis of the BP solution in neat ethylene glycol. Some lines of the two alcohol radicals are overlapped. Similar signals from the alcohol radicals were observed in experiments with uranyl and TEMPO, cf. Figure 3.

Addition of TEMPO $\left(5 \cdot 10^{-3}-2.5 \cdot 10^{-2} \mathrm{M}\right)$ to the solutions of BP in alcohols or their mixtures leads to the appearance of signals of polarized TEMPO ${ }^{\#}$, cf. Figure 7.

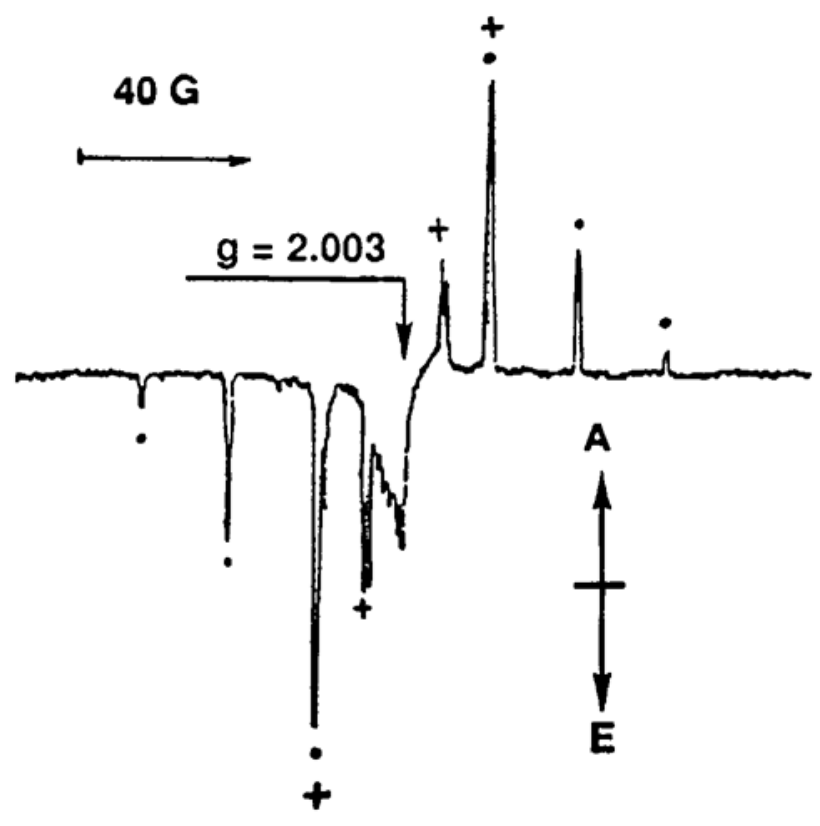

Figure 5. TR ESR spectrum obtained under photolysis of $\mathrm{BP}\left(1 \cdot 10^{-2} \mathrm{M}\right)$ in ethylene glycol/i-PrOH $(80: 20 \% \mathrm{v} / \mathrm{v})$ binary mixture; $\Delta \mathrm{t}=300-600 \mathrm{~ns}$. The dots stand for lines of $\left(\mathrm{CH}_{3}\right)_{2} \mathrm{C} \cdot \mathrm{OH}^{*}$; daggers denote lines of the $\mathrm{CH}_{2}(\mathrm{OH}) \mathrm{CH} \cdot \mathrm{OH}^{*}$ radical. 


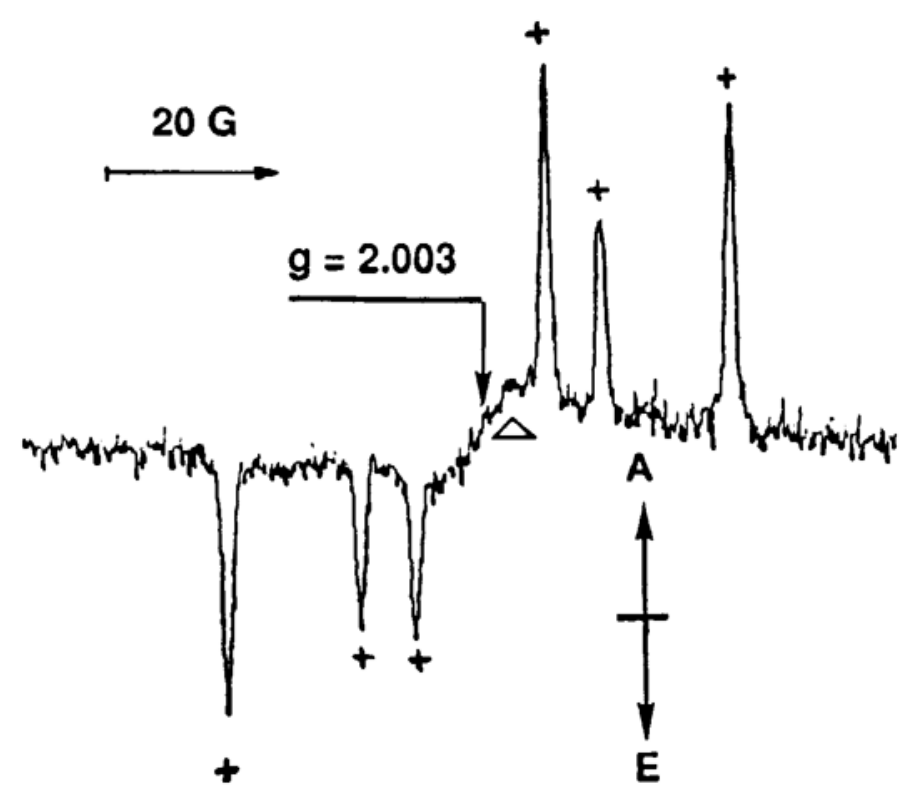

Figure 6. TR ESR spectrum obtained under photolysis of $\mathrm{BP}\left(1 \cdot 10^{-2} \mathrm{M}\right)$ in neat $n$ - $\mathrm{PrOH} ; \Delta \mathrm{t}=300-600$ ns. The daggers denote lines of $\mathrm{CH}_{3} \mathrm{CH}_{2} \mathrm{CH} \cdot \mathrm{OH}^{\#}$; triangles, the maximum absorption of $\mathrm{BPH}^{\# \text {. }}$.

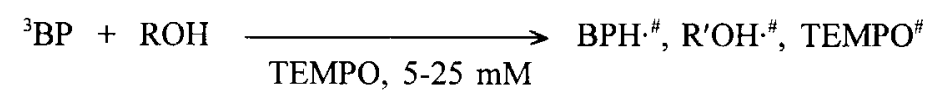

Under a relatively high concentration of TEMPO $\left(5 \cdot 10^{-2} \mathrm{M}\right.$ or more $)$ only the signals of TEMPO $^{4}$ were observed.

$$
{ }^{3} \mathrm{BP}+\mathrm{ROH} \underset{\mathrm{TEMPO}, \mathrm{C}>50 \mathrm{mM}}{\longrightarrow} \mathrm{TEMPO}^{*}
$$

A TR ESR study of the solutions was also performed in air-saturated solutions. Oxygen from the air has a relatively small effect leading to a decrease in TR ESR signals of $\mathrm{C}$-centred radicals.

\section{DISCUSSION}

CIDEP under the interaction of photoexcited triplet states and $r$

Photoexcited uranyl has many properties common to the triplet $n, \pi^{*}$ state of aromatic carbonyl compounds and, in particular, to the triplet state of benzophenone ${ }^{3} \mathrm{BP}[11,12]$. 


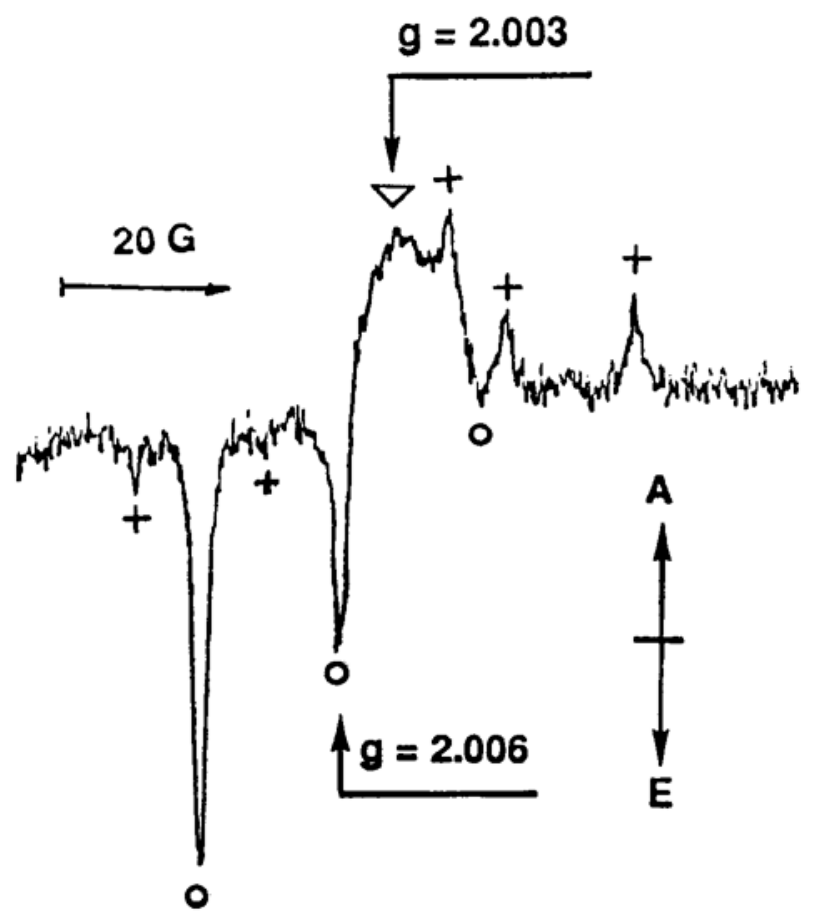

Figure 7. TR ESR spectrum obtained under photolysis of $\mathrm{BP}\left(1 \cdot 10^{-2} \mathrm{M}\right)$ in neat $n$-PrOH in the presence of TEMPO $\left(5 \cdot 10^{-3} \mathrm{M}\right) ; \Delta \mathrm{t}=300-600 \mathrm{~ns}$. The circles stand for lines of TEMPO ${ }^{\#}$; for the meaning of the other marks, see the caption to Figure 6.

Thus, it is convenient to begin the discussion with the quenching of ${ }^{3} \mathrm{BP}$ by $\mathbf{r}$, which is accompanied by the formation of CIDEP of $\mathbf{r}[2,3,5,13]$ :

$$
{ }^{3} \mathrm{BP}+\mathbf{r} \rightarrow{ }^{2.4}\left[{ }^{3} \mathrm{BP}, \mathbf{r}\right] \rightarrow \mathrm{BP}_{\mathrm{o}}+\mathbf{r}^{\#}
$$

It was reasonably proposed that emissive polarization of $\mathbf{r}^{\#}$ in reaction (10) is gained by a radical-triplet pair mechanism (RTPM) [2b-2d]. According to RTPM, the zero-field splitting (ZFS) modulated quartet-triplet interconversion in RTP in the region of non-zero exchange interaction $\mathbf{J}$ between doublet and quartet states of RTP leads to the emission of $\mathbf{r}^{\prime \prime}$ [2b-2d]. Interconversion of doublet and triplet states may also be affected by HFC, which can lead to HFC-dependence of polarized $\mathbf{r}^{\#}$, namely to a progressive decrease in the intensity of the high field components of $\mathbf{r}^{\sharp}$; the component in the highest field with $\mathrm{M}_{\mathrm{I}}=-1$ can result in absorption [2b-2d]. A more detailed description of RTPM can be found in references $[2 b-2 d, 14]$. 
CIDEP under the interaction of photoexcited uranyl nitrate with $\mathrm{TEMPO}^{+}$in solutions of NaPSS

The quenching of uranyl by TEMPO ${ }^{+}$or TEMPO ${ }^{+} \mathrm{NO}_{3}^{-}$in solutions of NaPSS leads to polarized TEMPO ${ }^{+\#}$, (Eq. (6)). CIDEP of $\mathbf{r}^{\#}$ was not observed in aqueous solutions, despite diffusion-controlled quenching of uranyl by $\mathbf{r}$ [4], but was observed only in the presence of a polyelectrolyte. It is known that uranyl is bound to anionic polyelectrolytes [15]. We propose that $\mathrm{TEMPO}^{+}$, as a compound with a positively charged tetramethylammonium group, is also concentrated in the vicinity of the polyanion [16]. Thus, the polyanion assists the creation (or transfer) of polarization under an interaction of uranyl and $\mathrm{TEMPO}^{+}$. In fact, according to RTPM, the magnitude of polarization is proportional to $\mathrm{D}^{2}$, where $\mathrm{D}$ is the zero field splitting parameter, and to solvent viscosity [2b,2d,14]. To our knowledge, D has never been measured for photoexcited uranyl. Measured values of ZFS of coordination compounds [17a] are usually smaller than those of organic compounds. In particular, ZFS of $\mathrm{Ru}(\mathrm{bpy})_{3}{ }^{2+*}$, showed a rather smaller than expected ZFS, namely $\mathrm{D}^{2} \cong 0.01 \mathrm{~cm}^{-2}[17 \mathrm{~b}]$, which is half that of ${ }^{3} \mathrm{BP}^{*}\left(\mathrm{D}^{2}=0.023 \mathrm{~cm}^{-2}\right.$ [18]). The relatively small $D^{2}$ of uranyl may be one of the reasons for the need for protracted contact of $\mathrm{UO}_{2}{ }^{2+*}$ with $\mathbf{r}$ for the creation of CIDEP. The strong effect of the binding of ionic reagents to polyelectrolytes in enhancement of photoinduced electron transfer and excited states quenching was clearly demonstrated in references [7,15-17,19]. The present results show that such binding facilitates the formation of CIDEP.

Coulombic binding of $\mathrm{TEMPO}^{+}$to negatively charged SDS micelles is less efficient in the present case and does not lead to CIDEP of TEMPO ${ }^{+}$, (Eq. (5)).

The iodide anion is known to be a very effective one-electron reductive quencher of photoexcited uranyl [20]. Nevertheless, polarized signals of $\mathrm{TEMPO}^{+} \mathrm{I}^{-}$have rather similar intensities to those of TEMPO ${ }^{+} \mathrm{NO}_{3}{ }^{-}$. The insignificant role of the counter anion of $\mathrm{TEMPO}^{+}$denotes that this anion is pushed out from the polyanion domain due to Coulombic repulsion.

At high concentrations, $\mathrm{TEMPO}^{+}$and $\mathrm{UO}_{2}{ }^{2+}$ compete for binding by the polyelectrolyte. At high $\left[\mathrm{UO}_{2}{ }^{2+}\right]$, which is comparable to the concentration of monomer units of NaPSS, the intensity of CIDEP decreases, reasons for which may be the ejection of $\mathrm{TEMPO}^{+}$from the vicinity of the polyanion, enhancement of self-quenching of uranyl in the polymer domain [15b], and the configuration changes of polymer structure under high concentration of the divalent cation, which can lead to stretching of the polymer and a decrease in the interaction of $\mathrm{UO}_{2}{ }^{2+*}$ with $\mathrm{TEMPO}^{+}$[7].

CIDEP under photolysis of BP and uranyl salts in solutions of alcohols and SDS micelles Photolysis of BP in most alcohols (Eq. (7)) leads to the expected E*/A CIDEP pattern (see Figure 5), which is explained by a radical pair mechanism (RPM) with a contribution of emissive spin polarization according to a triplet mechanism (TM) [10,21]. The observed CIDEP pattern in neat $i$-PrOH is the same as that presented in references $[10,21]$. 
The notable exception is CIDEP in solution in $n$-PrOH (Figure 6), which has an $\mathrm{E} / \mathrm{A}^{*}$ pattern. A similar problem, the appearance of inexplicable absorptive hyperfine coupling (HFC) independent polarization under the photolysis of acetone in $i$-PrOH and xanthone in ethanol, is under discussion in the current literature [22,23]. (We do not offer a consistent explanation of the reasons for an $\mathrm{E} / \mathrm{A}^{*}$ pattern of CIDEP in $n$-PrOH as well as for the two cited examples from the literature, and will only pay attention to the following.) The difference in properties of the two alcohols ( $i$ - $\mathrm{PrOH}$, expected $\mathrm{E}^{*} / \mathrm{A}$ pattern; $n$-PrOH, unexpected $\mathrm{E} / \mathrm{A}^{*}$ pattern) can be ascribed to the contribution of an electron transfer reaction in the latter case. The primary alcohols (ROH), which have higher $\alpha-\mathrm{C}-\mathrm{H}$ bond dissociation energies than those of the secondary alcohols, can be oxidized by one-electron oxidative agents into alkoxyl free radicals $\mathrm{RO}$. [23-25]. Alkoxyl radicals interact with the parent alcohol and convert it to $\mathrm{R}^{\prime} \mathrm{OH} \cdot$ [24]. Radicals $\mathrm{RO} \cdot$ have short relaxation times due to a $\mathrm{HFC}$-independent, spin-rotational relaxation. Such a relaxation proviso, not being extremely fast, might give rise to an absorptive contribution tot he CIDEP spectrum [22]).

Contrary to BP, photoexcited uranyl does not give rise to any CIDEP of photogenerated free radicals in all the solvents that were studied, nor to any CIDEP in micellar solutions. Oxidation of alcohols by triplet molecules leads to very noticeable signals of $\mathrm{R}^{\prime} \mathrm{OH} .{ }^{*}$ (e.g., see Figures 5 and 6); moreover, it is known that photoexcited uranyl oxidizes $\mathrm{BA}, \mathrm{BPH}_{2}$ and alcohols $\mathrm{ROH}$ with the formation of free radicals $[11,12]$. As an example,

$$
\mathrm{UO}_{2}^{2+^{+*}}+\mathrm{ROH} \rightarrow \mathrm{UO}_{2}^{+}+\mathrm{R}^{\prime} \mathrm{OH} \cdot+\mathrm{H}+
$$

The relatively short relaxation time of the metal-centred, uranoyl free radical $\mathrm{UO}_{2}{ }^{+}$in comparison with organic free radicals is not an inevitable reason for the absence of CIDEP. Thus, in photochemical reactions leading to the formation of triplet RPS consisting of organic metal-centred free radicals, the CIDEP signals of organic radicals were observed; whereas $\mathrm{Co}, \mathrm{Sn}$, and $\mathrm{Zn}$-centred free radicals did not show any signals due to their short paramagnetic relaxation times, $T_{1}[26,27]$.

To the best of our knowledge, the $\mathrm{T}_{1}$ for $\mathrm{UO}_{2}^{+}$has not been reported. It is difficult to suggest that an extremely fast paramagnetic relaxation of uranoyl is a possible reason for the absence of CIDEP. The ESR spectrum of uranoyl was observed at reduced [28] and at room temperatures [29]. A number of magnetic and spin effects in the photoreduction of uranyl by organic compounds, which proceeds from the formation of the RP containing uranoyl, were observed: CIDNP, magnetic field and magnetic isotope effects [13,30]. Furthermore, in a uranoyl free radical, the crystal field splitting parameter $\Delta\left(8000 \mathrm{~cm}^{-1}\right)$ is several times larger than the spin-orbit coupling (SOC) constant $\xi\left(2000 \mathrm{~cm}^{-1}\right)[28]$, which should not lead to a fast paramagnetic relaxation rate of this radical [31].

There may be a simple explanation, related to the structure of the uranoyl ion, for the absence of CIDEP in the organic radicals formed in photooxidation reactions. The uranoyl ion $\mathrm{UO}_{2}{ }^{2+}$ has a solvation shell of water molecules; when dissolved in organic 
solvent, it forms complexes in solution with organic molecules, which are coordinated in the equatorial plane of uranyl $[11,12]$. The high reactivity of $\mathrm{UO}_{2}{ }^{2+*}$ towards many organic substrates, and in particular alcohols, is related to the ability of $\mathrm{UO}_{2}{ }^{2+*}$ to oxidize a substrate either in an equatorial plane or by bimolecular attack by polar oxygen atoms of $\mathrm{UO}_{2}{ }^{2+*}$ with the formation of $\mathrm{UO}_{2}{ }^{+}$and an organic free radical [11,12]. Uranoyl $\mathrm{UO}_{2}{ }^{+}$ is a $5 \mathrm{f}^{1}$ uranium-centred free radical. There is no indication of delocalization of an unpaired electron to oxygen atoms of $\mathrm{UO}_{2}{ }^{+}[28]$, and $\mathrm{UO}_{2}{ }^{+}$and $\mathrm{UO}_{2}{ }^{2+}$ also have a solvation shell $[11,12]$.

CIDEP, according to a RPM mechanism, is formed under a "grazing" re-encounter of reagents, where the product of $|\mathbf{J}| \cdot \tau \cong 1$, where $\mathbf{J}$ is the exchange interaction for $R P$, and $\tau$ is the duration of such a contact [32]. the free radical of the substrate is formed, and then exits the solvation shell of uranoyl. Its place is promptly filled by solvent or substrate molecules, whose concentration is many orders of magnitude higher than that of organic free radicals.

Evidently, the re-encounter of an organic free radical with a $U$ atom, which bears the unpaired electron but is covered by the solvation shell, is a rare event, leading to low polarization coefficients or the absence of polarization. Such a specific contact of an atom $\mathrm{U}$ of $\mathrm{UO}_{2}^{+}$and free radical ("grazing" re-encounter) is not necessary for the occurrence of a redox reaction between these reagents, and uranoyl is known to have high or moderate reactivity to many organic free radicals [12b].

CIDEP under the photolysis of BP and uranyl salts in the presence of $r$ in solutions of alcohols

Photoexcitation of BP in alcohols in the presence of $\mathbf{r}$ (Eq. (8)), leads to polarized free radicals, see Figure 7 . The following elementary chemical reactions can occur in such solutions:

(i) photoreduction of ${ }^{3} \mathrm{BP}$ (Eq. (9)), which can lead to RPM and TM polarization of radicals $\left(\mathrm{E} / \mathrm{A}^{*}\right.$ or $\mathrm{E}^{*} / \mathrm{A}$ - pattern);

(ii) quenching of ${ }^{3} \mathrm{BP}$ (Eq. (7)) by radicals, which should lead to RTPM polarization of $r$ with $E+E / A$ pattern;

(iii) creation of polarization in random encounters ( $F$-pairs) of reactive radicals, which should lead to an E/A - pattern (or $E^{*} / A$ pattern of CIDEP of one and E/A* pattern of another radical [32]).

$$
\begin{aligned}
\mathrm{R}^{\prime} \mathrm{OH} \cdot+\mathbf{r} & \rightarrow \mathrm{R}^{\prime} \mathrm{OH}^{*}+\mathbf{r}^{\#} \\
\mathrm{BPH} \cdot+\mathbf{r} & \rightarrow \mathrm{BPH}^{*}+\mathbf{r}^{*}
\end{aligned}
$$

(iv) transfer of net polarization (if it exists) from free radicals to TEMPO, which should lead to E- or A-pattern of CIDEP of $\mathbf{r}^{\#}$ :

$$
\mathrm{R}^{\prime} \mathrm{OH}^{*} \cdot+\mathbf{r} \rightarrow \mathrm{R}^{\prime} \mathrm{OH}+\mathbf{r}^{*}
$$




$$
\mathrm{BPH}^{\#} \cdot+\mathbf{r} \rightarrow \mathrm{BPH} \cdot+\mathbf{r}^{\#}
$$

Equations 11a and 12a probably do not contribute (or make only a minor contribution) to the observed CIDEP of $\mathbf{r}$. In fact, the CIDEP spectrum $\mathrm{R}^{\prime} \mathrm{OH}$. and $\mathrm{BPH} \cdot$ has the E/A* pattern with the contribution of HFC-independent absorptive polarization (Figure 6), whereas the same system with the presence of $\mathbf{r}$, the emissive polarization of $\mathbf{r}^{*}$ was observed ( $E^{*} / A$ pattern, Figure 7). This means that the absorptive polarization is not effectively transferred, or that it makes a smaller contribution to the observed polarization than the emissive polarization of $\mathbf{r} \#$.

Only Eqs. (11) and (12) cannot lead to the observed E*/A pattern of CIDEP spectrum of $\mathbf{r}$, the observed CIDEP. It is evident that the high-field component of TEMPO should be in absorption under the action of RPM in F-pairs [32], and the computer simulated CIDEP spectrum of TEMPO participating in Eqs. (11) and (12) confirms this expectation. The observed spectrum of TEMPO ${ }^{\#}$ shows the high-field component in emission. Thus, we must admit that Eq. (10) produces the largest contribution to the CIDEP of TEMPO.

There is an appreciable difference between the g-factors of TEMPO ( $g=2.006$ [9]) and those of $\mathrm{R}^{\prime} \mathrm{OH}$ and $\mathrm{BPH}$ (both have $\mathrm{g}=2.003$ [9]). This difference should result in a E/A* - pattern of CIDEP of radicals $\mathrm{CH}_{3} \mathrm{CH}_{2} \mathrm{CH} \cdot \mathrm{OH}$ and $\mathrm{BPH}$, and such a polarization was observed, $c f$. Figure 7. Photoreduction (Eq. (7)) of BP in the $n$-PrOH reaction leads to the same polarization pattern $\left(E / A^{*}\right)$, see above. Finally we admit that Eqs. (7), (11) and (12) lead to observed CIDEP (Figure 7) of $\mathrm{BPH} \cdot$ and $\mathrm{CH}_{3} \mathrm{CH}_{2} \mathrm{CH} \cdot \mathrm{OH}$.

Under a high concentration of $\mathbf{r}$ only polarized $\mathbf{r}^{\sharp}$ is observed, Eq. (9). There are two reasons for such an observation. First, the higher the concentration of $\mathbf{r}$, the larger the contribution of quenching reaction 10 and the smaller the contribution of photoreduction, reaction 7. Second, under a high concentration of $\mathbf{r}$ radicals of $\mathrm{R}^{\prime} \mathrm{OH}$. and $\mathrm{BPH} \cdot$, if they form, will be trapped:

$$
\begin{aligned}
& \mathrm{R}^{\prime} \mathrm{OH}^{(*)}+\mathbf{r} \underset{\mathbf{r}>50 \mathrm{mM}}{\longrightarrow} \text { No CIDEP } \\
& \mathrm{BPH}^{(*)}+\mathbf{r} \underset{\mathbf{r}>50 \mathrm{mM}}{\longrightarrow} \text { No CIDEP }
\end{aligned}
$$

The more reactive radical $\mathrm{R}^{\prime} \mathrm{OH}$ is supposed to react faster with $\mathbf{r}$ than $\mathrm{BPH}$. This is the probable reason for the relative increase in intensity of the observed signals of $\mathrm{BPH}^{. \sharp}$ with respect to that of $\mathrm{R}^{\prime} \mathrm{OH} \cdot{ }^{\sharp}$ in the presence of $\mathbf{r}$ ( $c f$. Figures 6 and 7). In other words, when polarized radicals are generated in reaction (7), a larger fraction of $\mathrm{R}^{\prime} \mathrm{OH} \cdot{ }^{*}$ than of $\mathrm{BPH}^{*}$ is quenched in the reaction with $\mathbf{r}$.

The interaction of photoexcited uranyl in solvents of moderate viscosity leads to CIDEP signals of $\mathbf{r}^{\prime \prime}$ : 


$$
\mathrm{UO}_{2}{ }^{2+*}+\mathbf{r} \rightarrow \mathrm{UO}_{2}{ }^{2+}+\mathbf{r}^{*}
$$

cf. Figures 1 and 2. The interaction between reagents is enhanced due to increased solvent viscosity (vide supra), and the RTPM seems to be operative. The decrease in the intensity of HF components of $\mathbf{r}^{\#}$ with a decrease in $M_{I}$ is also a vestige of RTPM (see the first Discussion section, above). The probable reason for the absence of signals of $\mathbf{r}^{\#}$ in solvents of higher viscosity (e.g., cyclohexanol, $\eta=\sim 45 \mathrm{cP}$ ) is retardation of mutual diffusion and the low rate of polarization creation in reaction (10a). Photoexcited uranyl also oxidizes cyclohexanol and other viscous solvents (reaction (la)), and CIDEP of $\mathbf{r}$ might be formed in reaction (11a). However, this is not the case, probably due to the same reason, i.e. retardation of mutual diffusion as well as enhanced cage effect values in reaction (1a).

\section{CONCLUSIONS}

The nominate triplet multiplicity is assigned to photoexcited uranyl being based on a set of experimental data and theoretical considerations [11a,12]. (The adjective "nominate" stands for the fact that the triplet state is not a pure spin state and has an admixture of the singlet state). Photoexcited uranyl is effectively quenched by $\mathbf{r}$, so it can be expected that such quenching will lead to emissive signals of $\mathbf{r}$ as in other triplet states due to the action of RTPM and the usual suggestion of a negative sign of the exchange integral, $\mathbf{J}$. These signals were observed in NaPSS solutions, where one can expect the effective interaction of two cations assisted by a polyanion as well as in solvents of moderate viscosity $(\eta=3-10 \mathrm{cP})$.

Photoexcited uranyl salts oxidize many compounds including alcohols with the production of corresponding free radicals $[11,12]$. However, we did not find any CIDEP signals of these radicals. Such behaviour distinguishes $\mathrm{UO}_{2}{ }^{2+*}$ from its organic analog, triplet benzophenone. Photooxidation by benzophenone leads to strong CIDEP signals of radicals of alcohols and of other donors. The probable reason for the lack of polarization in uranyl photoreduction reactions is the difficult access of free radicals to the $\mathrm{U}$ atom of the solvated $\mathrm{UO}_{2}^{+}(\mathrm{V})$. This atom bears the unpaired electron. At the same time, free radicals photoinduced by uranyl can gain CIDEP in F-pairs interacting with stable nitroxyl radicals added in moderate concentration $\left(5 \cdot 10^{-3}-1 \cdot 10^{-2} \mathrm{M}\right)$. Under higher concentrations of nitroxyls, the uranyl's photoexcited state is effectively quenched and/or most of its reactive radicals are trapped, and no CIDEP is observed in non-viscous solvents.

\section{Acknowledgements}

The authors thank the NSF, DOE and AFOSR for their generous support of this research. I.V.K. thanks Dr. Yu. A. Serebrennikov for fruitful discussion and comments. 


\section{REFERENCES}

1. V.A. Kuzmin and A.S. Tatikolov, Chem. Phys. Lett. $\underline{\mathbf{5 3}} 606$ (1978).

2. a) T. Imamura, O. Onitsuka and K. Obi, J. Phys. Chem. 90, 6741 (1986); b) A. Kawai, T. Okutsu and K. Obi, J. Phys. Chem. 95, 9130 (1991); c) A. Kawai and K. Obi, J. Phys. Chem. 96, 52 (1992); d) A. Kawai and K. Obi, J. Phys. Chem. 96, 5701 (1992).

3. W.S. Jenks and N.J. Turro, Res. Chem. Intermed. 13, 237 (1990).

4. A.P. Darmanyan and I.V. Khudyakov, Photochem. Photobiol. 52, 293 (1990).

5. W.S. Jenks, Ph. D. Thesis, Columbia University, New York, New York, 1991.

6. N.J. Turro and I.V. Khudyakov, Chem. Phys. Lett. 193, 546 (1992).

7. D. Meisel, J. Rabani, D. Meyerstein and M.S. Matheson, J. Phys. Chem. 82, 985 (1978).

8. J. Timmermans. The Physico-chemical Constants of Binary Systems in Concentrated Solutions. Interscience, New York (1959).

9. H. Fischer and K.-H. Helwege (Eds.). Magnetic Properties of Free Radicals. Springer, Berlin (1977).

10. A.D. Trifunac, M.C. Thurnauer and J.R. Norris, Chem. Phys. Lett. 57, 471 (1978).

11. a) H.D. Burrows and T.J. Kemp, Chem. Soc. Rev. 3, 139 (1974); b) H.D. Burrows, S.J. Formosinho, F. Pinto Coelho, M.G.M. Miguel and M.E.D.G. Azenha, Mem. Acad. Ciencias Lisboa 30,33 (1989).

12. a) A.L. Buchachenko and I.V. Khudyakov, Accounts Chem. Res. 24, 177 (1991); b) A.L. Buchachenko and I.V. Khudyakov, Usp. Khimii 60, 1105 (1991) [Russian Chem. Rev. 60, 555 (1991)].

13. N.J. Turro, I.V. Khudyakov, S.H. Boßmann and D.W. Dwyer, J. Phys. Chem. submitted for publication.

14. a) C. Blättler, F. Jent and H. Paul, Chem. Phys. Lett. 166, 375 (1990); b) C. Blättler and H. Paul, Res. Chem. Intermed. 16, 201 (1991).

15. a) T. Rosenfeld-Grü nwald and J. Rabani, J. Phys. Chem. 84, 2981 (1980); b) I.A. Taha and H. Morawetz, J. Am. Chem. Soc. 93,829 (1971).

16. T. Okubo and N.J. Turro, J. Phys. Chem. 85, 4034 (1981).

17. a) A.L. Kamyshny, A.P. Suisalu and L.A. Aslanov, Coord. Chem. Rev. 117, 1 (1992); b) S. Yamauchi, Y. Komada and N. Hirota, Chem. Phys. Lett. 129, 197 (1986).

18. a) H. Murai, T. Imamura and K. Obi, Chem. Phys. Lett. 87, 295 (1982); b) K. Miyagawa, H. Murai and Y.J. I'Haya, Chem. Phys. Lett. 118, 140 (1985).

19. N.J. Turro, J.K. Barton and D.A. Tomalia, Accounts Chem. Res. 24, 332 (1991).

20. H.D. Burrows, Inorg. Chem. 29, 1549 (1990).

21. H. Hayashi, Y. Sakagushi, H. Murai and Y.J. I'Haya, J. Phys. Chem. 90, 4403 (1986).

22. K.A. McLauchlan, N.J.K. Simpson and P.D. Smith, Res. Chem. Intermed. 16, 141 (1991).

23. H. Murai and K. Kuwata, J. Phys. Chem. 95, 6247 (1991).

24. C.R.H.I. de Jonge, I.V. Khudyakov, S.L. Krenyov, V.A. Kuzmin, A.I. Prokof'ev and M.V. Voevodskaya, J. Chem. Soc. Perkin Trans. 2, 347 (1985).

25. V.M. Kuznetz, P.P. Levin, I.V. Khudyakov and V.A. Kuzmin, Izvestia AN SSR. Ser. Khim. 1284 (1978).

26. a) P. Levinstein and $H$. van Willigan, Chem. Phys. Lett. 187, 415 (1991); b) P. Levinstein, M. Ebersol and F.W. Pijpers, Mol. Cryst. Liquid Cryst. 194, 123 (1991).

27. Y. Sakaguchi, H. Hayashi and Y.J. I'Haya, J. Phys. Chem. 94, 291 (1990).

28. C. Miyake, Y. Yamana, S. Imoto and H. Ohya-Nishiguchi, Inorg. Chim. Acta 95, 17 (1984).

29. a) S.S. Sandhu, M.S. Sidhu and R.J. Singh, J. Photochem. Photobiol A39, 229 (1987); b) S.S. Sandhu, M.S. Sidhu and R.J. Singh, J. Photochem. Photobiol. A46, 213 (1989).

30. S.V. Rykov, I.V. Khudyakov, E.D. Skakovski, L. Yu. Tychinskaya and M.M. Ogorodnikova, J. Photochem. Photobiol. A66, 127 (1992).

31. a) Yu. A. Serebrennikov and U.E. Steiner. Magnetic and Spin Effects in Chemistry and Related Phenomena. Abstr. of Intern. Symp. on Magn. Eff., University of Konstanz, Konstanz, Germany, 
July $26-31,1992$, p. 53; b) I.V. Khudyakov, Yu. A. Serebrennikov and N.J. Turro, Chem. Rev. submitted for publication.

32. a) F.J. Adrian, Res. Chem. Intermed. 16, 99 (1991); b) F.J. Adrian, Rev. Chem. Intermed. $\underline{7}, 173$ (1986); c) K.A. McLauchlan. In: Lasers in Polymer Science and Technology: Applications, J.-P. Fouassier and J.F. Rabek (Eds.). vol. 1, p. 259. CRC Press, (1990). 